\title{
EXCEPTIONAL COVERS OF SURFACES
}

\author{
JEFFREY D. ACHTER
}

\begin{abstract}
Consider a finite morphism $f: X \rightarrow Y$ of smooth, projective varieties over a finite field $\mathbb{F}$. Suppose $X$ is the vanishing locus in $\mathbb{P}^{N}$ of $r$ forms of degree at most $d$. We show that there is a constant $C$ depending only on $(N, r, d)$ and $\operatorname{deg}(f)$ such that if $|\mathbb{F}|>C$, then $f(\mathbb{F}): X(\mathbb{F}) \rightarrow Y(\mathbb{F})$ is injective if and only if it is surjective.
\end{abstract}

\section{INTRODUCTION}

Consider a finite, generically étale morphism $f: X \rightarrow Y$ between smooth, projective varieties over a finite field $\mathbb{F}$ of characteristic $p$. The cover $f$ is called exceptional if the only geometrically irreducible component of $X \times_{Y} X$ which is defined over $\mathbb{F}$ is the diagonal. Exceptional covers have the following intriguing property: the induced map $f(\mathbb{F}): X(\mathbb{F}) \rightarrow Y(\mathbb{F})$ on $\mathbb{F}$-points is bijective. This theorem, due to Lenstra, is proved in [6]; we defer to that article for the history of this circle of ideas.

In [6], Guralnick, Tucker and Zieve prove a partial converse for projective curves. Specifically, they show that for fixed genus $g=g(X)$ and degree $\operatorname{deg}(f)$, there exists an effective constant $C$ such that the following holds: if $\mathbb{F}_{q} / \mathbb{F}$ is an extension with $q>C$, and if $f\left(\mathbb{F}_{q}\right)$ is injective, then $f$ is exceptional. (Note that this implies that $f$ is bijective.) They prove something like this in higher dimension (see Remark 2.6 below), except that the constant $C$ is allowed to depend on $X, Y$ and $f$. They conjecture [6,5.5] that $C$ need only depend on $\operatorname{deg}(f)$ and the topology of $X$.

The calculation of $C$ relies on understanding the topology of the cover $f$. Indeed, if $Z$ is a nondiagonal component of $X \times_{Y} X$, and if $f\left(\mathbb{F}_{q}\right)$ is injective, then every point of $Z\left(\mathbb{F}_{q}\right)$ is actually a ramification point. The dimension of $Z$ is greater than that of the ramification locus $\operatorname{ram}(f)$. Weil-type estimates show that if $\left|Z\left(\mathbb{F}_{q}\right)\right| \leq\left|\operatorname{ram}(f)\left(\mathbb{F}_{q}\right)\right|$, then $q$ must be small relative to the Betti numbers of $Z$ and $\operatorname{ram}(f)$. In the special case of curves, Guralnick, Tucker and Zieve obtain effective bounds for these Betti numbers, and thus a bound for the constant $C$.

Consider the following condition on a triple $(X / \mathbb{F}, C, n)$ consisting of a smooth, projective, geometrically irreducible variety $X$, a constant $C$, and a natural number $n \geq 2$ :

Condition 1.1. Let $Y / \mathbb{F}$ be a smooth projective geometrically irreducible variety, and let $f: X \rightarrow Y$ be a finite, tamely ramified, generically étale morphism of degree $n$. Then $(f: X \rightarrow Y / \mathbb{F}, C)$ satisfies (*):

If $\mathbb{F}_{q} / \mathbb{F}$ is a finite extension with $q>C$, then the following are equivalent:

(*)
(a) $f\left(\mathbb{F}_{q}\right): X\left(\mathbb{F}_{q}\right) \rightarrow Y\left(\mathbb{F}_{q}\right)$ is injective;
(b) $f\left(\mathbb{F}_{q}\right): X\left(\mathbb{F}_{q}\right) \rightarrow Y\left(\mathbb{F}_{q}\right)$ is surjective;
(c) $f_{\mathbb{F}_{q}}: X_{\mathbb{F}_{q}} \rightarrow Y_{\mathbb{F}_{q}}$ is exceptional.

2000 Mathematics Subject Classification. 11G25. 
The main purpose of this note is to explain the following result. Say that a projective variety $X$ is of type $(N, r, d)$ if $X$ is isomorphic to the vanishing in $\mathbb{P}^{N}$ of at most $r$ homogeneous forms, each of which has degree at most $d$.

Theorem 2.5. Given data $(N, r, d)$ and $n$, there exists a constant $C$ so that the following holds. If $X$ is a smooth, projective geometrically irreducible of type $(N, r, d)$, then $(X / \mathbb{F}, C, n)$ satisfies (1.1).

The type $(N, r, d)$ is not intrinsic to $X$, but is rather an attribute of $X$ along with a chosen embedding. In fact, [6, Conjecture 5.5] asks for a result similar to 2.5, but which depends only on the $\ell$-adic Betti numbers of the source variety. Here is a partial answer for surfaces:

Corollary 3.2. Given nonnegative integers $b_{1}, b_{2}$ and $b_{3}$ and a natural number $n$, there exists an effective constant $C$ so that the following holds. Let $X$ be a smooth, projective, geometrically irreducible surface over a finite field $\mathbb{F}$. Suppose that $X$ is of general type and admits a flat lifting to $W(\mathbb{F}) / p^{2}$, and that $\operatorname{dim} H^{i}\left(X, \mathbb{Q}_{\ell}\right)=b_{i}$ for $1 \leq i \leq 3$. Then $(X / \mathbb{F}, C, n)$ satisfies (1.1).

Results such as Theorem 2.5 have long been known in the special case where $X=Y=\mathbb{P}^{n}$ [5]. For arbitrary varieties, [6. Prop. 5.6] shows the existence of a constant $C$ which depends on $f: X \rightarrow Y$ such that $(f: X \rightarrow Y / \mathbb{F}, C)$ satisfies $\left.{ }^{*}\right)$. The contribution of the present note is to prove a more uniform version of these results.

The final section gives a new supply (Proposition 4.1) of examples of exceptional covers, as well as examples of covers which are injective or surjective on $\mathbb{F}$-points, but not bijective. In contrast to previously published examples, which tend to focus on curves, projective spaces or abelian varieties, these covers involve varieties of arbitrary dimension and arbitrarily intricate topology.

I thank Guralnick for a helpful discussion of [6, Prop. 5.6]; see Remark 2.6.

\section{EXCEPTIONAL COVERS OF POLARIZED VARIETIES}

Say that a projective variety $X$ admits a polarization of type $(N, r, d)$, or simply that $X$ has type $(N, r, d)$, if there exists an embedding $X \hookrightarrow \mathbb{P}^{N}$ such that $X$ can be expressed as the vanishing locus of at most $r$ homogeneous forms, each of which has degree at most $d$. Similarly, say that an affine variety $U$ has type $(N, r, d)$ if there exists an embedding $U \hookrightarrow \mathbb{A}^{N}$ such that $U$ is the vanishing locus of at most $r$ polynomials, each of which has degree at most $d$.

Suppose $X$ is projective of type $(N, r, d)$, and that $X \hookrightarrow \mathbb{P}^{N}$ realizes this type. If $H \subset \mathbb{P}^{N}$ is a hyperplane, then $X \cap H$ is projective of type $(N-1, r, d)$, while $X-(X \cap H)$ is affine of type $(N, r, d)$. For the moment, we work over an arbitrary and suppressed field $k$.

Lemma 2.1. Gven data $(N, r, d)$ and $n$ there exist effective constants $(\widetilde{N}, \widetilde{r}, \widetilde{d})$ such that the following holds. If $X$ is a geometrically irreducible projective variety of type $(N, r, d)$, and if $f: X \rightarrow Y$ is a finite map of degree $n$, then $Y$ is projective of type $(\widetilde{N}, \widetilde{r}, \widetilde{d})$.

Proof. Embed $Y \hookrightarrow \mathbb{P}^{M}$, where $M=2 \operatorname{dim} Y+1$. On an open affine subset $U \subseteq X, f$ is represented by a map $\left(f_{0}, \cdots, f_{M}\right)$, with each $f_{j} \in k\left[\mathbb{A}^{N}\right]$. By Bézout's theorem, each $f_{j}$ has degree at most $n$. Elimination theory, either in its classical form or its Gröbner-theoretic incarnation, yields an effective upper bound on the number and degree of the equations necessary to define the Zariski closure of $f(U)$ in $\mathbb{A}^{M}$. 
Lemma 2.2. Given data $(N, r, d)$ and $n$ there are effective constants $\left(N_{\mathrm{r}}, r_{\mathrm{r}}, d_{\mathrm{r}}\right)$ and $\left(N_{\mathrm{b}}, r_{\mathrm{b}}, d_{\mathrm{b}}\right)$ such that the following holds. If $X$ is a geometrically irreducible projective variety of type $(N, r, d)$ and if $f: X \rightarrow Y$ is a finite map of projective varieties of degree $n$, then the ramification locus $\operatorname{ram}(f) \subset X$ is of type $\left(N_{\mathrm{r}}, r_{\mathrm{r}}, d_{\mathrm{r}}\right)$, and the branch locus branch $(f) \subset Y$ is of type $\left(N_{\mathrm{b}}, r_{\mathrm{b}}, d_{\mathrm{b}}\right)$.

Proof. As in the proof of Lemma 2.1, represent $f$ locally by a morphism $\left(f_{1}, \cdots, f_{M}\right): \mathbb{A}^{N} \rightarrow \mathbb{A}^{M}$, and let $J f=\left(\frac{\partial f_{i}}{\partial x_{j}}\right)_{1 \leq i \leq M, 1 \leq j \leq N}$ be the Jacobian matrix of this morphism. The ramification locus $\operatorname{ram}(f)$ is the locus of all $P \in U$ where the restriction of $J f$ to $T_{P} U$ has rank less than $n$. Therefore, $\operatorname{ram}(f)$ is the intersection of $U$ and the vanishing locus of minors of a certain matrix, and its type $\left(N_{\mathrm{r}}, r_{\mathrm{r}}, d_{\mathrm{r}}\right)$ depends only on the type of $X$ and on the degree of $f$.

Lemma 2.1, applied to the cover $\operatorname{ram}(f) \rightarrow \operatorname{branch}(f)$, shows that branch $(f)$ is of type $\left(N_{\mathrm{b}}, r_{\mathrm{b}}, d_{\mathrm{b}}\right)$ for constants which depend only on $(N, r, d, n)$.

Let $g: Z \rightarrow Y$ be an étale cover of affine varieties. Say that $g$ is tamely ramified at the boundary if there are compactifications $Z \hookrightarrow \bar{Z}$ and $Y \hookrightarrow \bar{Y}$, with $\bar{Z}$ and $\bar{Y}$ projective, and a morphism $\bar{g}: \bar{Z} \rightarrow \bar{Y}$ compatible with $g$ which is at worst tamely ramified. (This notion is independent of the choice of compactification; moreover, in the present context, the cover $g: Z \rightarrow Y$ arises as an open subcover of a known cover of projective varieties.)

Lemma 2.3. Given data $(N, r, d)$ there exists an effective constant $\sigma$ such that the following holds. Let $Y$ be a smooth geometrically irreducible affine variety of type $(N, r, d)$ over a field $k$, and let $g: Z \rightarrow Y$ be an étale Galois cover tamely ramified at the boundary. If $\ell$ is any rational prime invertible in $k$, then the sum of the compact $\ell$-adic Betti numbers of $Z$ satisfies

$$
\sigma_{c}\left(Z, \mathbb{Q}_{\ell}\right):=\sum_{i=0}^{\operatorname{dim} Z} \operatorname{dim} H_{c}^{i}\left(Z \times \bar{k}, \mathbb{Q}_{\ell}\right) \leq \operatorname{deg}(g) \sigma .
$$

Proof. This is [7, Prop. 4.5]; the effective constant of loc. cit. depends only on the type of $Y$.

Lemma 2.4. Given data $(N, r, d)$ and $n$ there exist effective constants $\left(N_{\mathrm{u}}, r_{\mathrm{u}}, d_{\mathrm{u}}\right)$ such that the following holds. Let $X$ be a smooth geometrically irreducible projective variety of type $(N, r, d)$, and let $f: X \rightarrow Y$ be a finite morphism of degree $n$ which is generically étale. Then there is an open subvariety $U \subset Y$, affine of type $\left(N_{\mathrm{u}}, r_{\mathrm{u}}, d_{\mathrm{u}}\right)$, such that $\left.f\right|_{f^{-1}(U)}$ is étale.

Proof. By Lemmas 2.1 and 2.2, there is a collection of homogeneous forms $\left\{G_{1}, \cdots, G_{s}\right\}$ of bounded degree on $Y$ such that $f$ is unramified outside the vanishing locus of these forms. Let $U$ be the complement of the vanishing locus of the product $\prod G_{i}$. Then $\left.f\right|_{f^{-1}(U)}$ is étale, and $U$ has known type $\left(N_{\mathrm{u}}, r_{\mathrm{u}}, d_{\mathrm{u}}\right)$.

Theorem 2.5. Given data $(N, r, d)$ and $n$ there exists an effective constant $C$ so that the following holds. Let $X / \mathbb{F}$ be a smooth geometrically irreducible projective variety of type $(N, r, d)$. Then $(X / \mathbb{F}, C, n)$ satisfies (1.1).

Proof. We adopt the notation, ideas and results of [6]. Say that a variety is of known type if its type can be effectively bounded purely in terms of the data $(N, r, d, n)$. Let $Y / \mathbb{F}$ be a smooth geometrically irreducible projective variety, and let $f: X \rightarrow Y$ be a finite, tamely ramified, generically étale morphism of degree $n$. By Lemma2.1, $Y$ is of known type. 
Let $\widetilde{X} \rightarrow Y$ be the Galois closure of $X \rightarrow Y$. Then $\widetilde{X}$ is a variety over some finite extension $\widetilde{\mathbb{F}}$ of $\mathbb{F}$, and there is a finite map of schemes $\widetilde{f}: \widetilde{X} \rightarrow Y$ of degree $\widetilde{n} \leq n$ ! which is tamely ramified and generically étale. Let $A=\operatorname{Aut}(\widetilde{X} / Y)$, and let $G=\operatorname{Aut}\left(\widetilde{X} / Y \times \widetilde{\mathbb{F}}_{q}\right)$ be the geometric part of the extension; then $A / G \cong \operatorname{Gal}(\widetilde{\mathbb{F}} / \mathbb{F})$. Let $H=\operatorname{Aut}(\widetilde{X} / X)$, let $\mathcal{S}$ be the set of left cosets of $H$ in $A$, and let $\mathcal{B}=\{a \in A:\langle a G\rangle=A / G\}$. By [6, Lemmas 4.2 and 4.3], to show that $f$ is exceptional it suffices to show that each $a \in \mathcal{B}$ has at least one fixed point in $S$.

Using Lemma 2.4, we may construct an affine subvariety $U \subset Y$ of known type such that $\left.f\right|_{f^{-1}(U)}$ is unramified. Let $\widetilde{V}=\widetilde{X} \times U$; then $\left.\widetilde{f}\right|_{\widetilde{V}}: \widetilde{V} \rightarrow U$ is an étale Galois cover of affine varieties. Let $\ell$ be any rational prime invertible in $\mathbb{F}$. Lemma 2.3 gives an effective upper bound for the sum of the compact Betti numbers $\sigma_{c}\left(\widetilde{V}, \mathbb{Q}_{\ell}\right)$.

Let $\widetilde{W}$ be any twist of $\widetilde{V}$. Then $\sigma_{c}\left(\widetilde{W}, \mathbb{Q}_{\ell}\right)=\sigma_{c}\left(\widetilde{V}, \mathbb{Q}_{\ell}\right)$. By the Lefschetz trace formula [2, II.3.2] and Deligne's bound for the weights of Frobenius on the compact cohomology groups of a smooth variety [3, Thm. 1], we find that there is an effective constant $C$, depending only on $(N, r, d, n)$, such that if $\mathbb{F}_{q} / \mathbb{F}$ is a finite field, and if $q>C$, then $\widetilde{W}\left(\mathbb{F}_{q}\right)$ is nonempty.

The result now follows from the techniques of [6]. Fix any $a \in \mathcal{B}$. Restricting the construction of [6, paragraph after 3.2] to $\widetilde{V}$, construct a certain twist $\widetilde{V}^{\tilde{a}}$ of $\widetilde{V}$. If $q>C$, then there exists some $\widetilde{Q}_{a} \in \widetilde{V}^{\widetilde{a}}\left(\mathbb{F}_{q}\right)$. Let $P_{a}=f\left(\widetilde{Q}_{a}\right)$. Combining the defining property of $\widetilde{V}^{\widetilde{a}}$ with the fact that $\left.\widetilde{f}\right|_{\widetilde{V}}$ is unramified, we have $\widetilde{Q}_{a} / P_{a}$ is unramified, with decomposition group $\langle a\rangle$. Moreover, for this point $P_{a}$, the number of points of $X\left(\mathbb{F}_{q}\right)$ lying over $P_{a}$ is exactly the number of points of $\mathcal{S}$ fixed by $a$, 6 , Lemma 3.2]. Henceforth, suppose $q>C$.

If $f$ is surjective on $\mathbb{F}_{q}$ points, then for each $a \in \mathcal{B}$ there is at least one point of $X\left(\mathbb{F}_{q}\right)$ lying over $P_{a}$, so that at least one point of $\mathcal{S}$ is fixed by $a$. Then $f$ is exceptional [6, Lemma 4.3].

Similarly, if $f$ is injective on $\mathbb{F}_{q}$ points, then for each $a \in \mathcal{B}$ there is at most one point of $\mathcal{S}$ fixed by $a$, and $f$ is again exceptional.

Finally, Lenstra's theorem [6, Prop. 4.4] shows that if $f$ is exceptional, then it is in fact bijective on $\mathbb{F}_{q}$-points.

Remark 2.6. Even though this statement depends on a polarization of the variety $X$, it is still much more uniform than the best result previously known. For comparison's sake, note that [6, Prop. 5.6] states that given $f: X \rightarrow Y$ a finite separable map between normal varieties over $\mathbb{F}$, if $f\left(\mathbb{F}_{q^{m}}\right)$ is injective or surjective for infinitely many $m$, then $f$ is exceptional. As noted there, this implies the existence of a constant $C$, depending on $f: X \rightarrow Y$, such that $(f: X \rightarrow Y, C, \mathbb{F})$ satisfies $\left(^{*}\right)$. Indeed, the proof of loc. cit. shows the existence of a number $M$ such that if $m \geq M$, then the surjectivity or injectivity of $f\left(\mathbb{F}_{q^{m}}\right)$ implies the exceptionality of $f_{\mathbb{F}^{m}}$.

Remark 2.7. If $X$ is a member of a known family then Theorem 2.5 provides a uniform bound for $C$, in the following sense.

Suppose $S$ is noetherian and $\mathcal{X} \rightarrow S$ is has geometric fibers which are smooth, projective and irreducible. Then there exist $\left(N_{S}, r_{S}, d_{S}\right)$ such that for any point $t \in S(\mathbb{F})$, the fiber $\mathcal{X}_{t}$ has type $\left(N_{S}, r_{S}, d_{S}\right)$. Consequently, for any $n$ there exists an effective constant $C=C(S, n)$ such that if $t \in S(\mathbb{F})$, then $\left(\mathcal{X}_{t} / \mathbb{F}, C, n\right)$ satisfies (1.1).

Natural examples of such families $\mathcal{X} \rightarrow S$ are the tautological families over (an open subscheme of) the Hilbert scheme of schemes of $\mathbb{P}^{N}$ with specified Hilbert polynomial; the moduli space of principally polarized abelian varieties of given dimension; and the moduli space of $\mathrm{K} 3$ surfaces 
with polarization of specified degree. In fact, many moduli spaces are constructed by taking the GIT quotient of an open subscheme $S$ of a Hilbert scheme. While the difficulties typically center around the construction of the quotient space, the techniques of the present paper apply directly to $S$.

\section{SURFACES}

If the varieties $X$ and $Y$ are curves, then [6, Thm. 4.7] gives an explicit bound for the constant $C$ of 2.5 which depends only on $n=\operatorname{deg}(f)$ and the genus of $X$. In this section, we show that if $X$ is a surface of general type, then there is a constant $C$ which depends only on the Hodge numbers of $X$ and on $n$ such that $(X / \mathbb{F}, C, n)$ satisfies (1.1). If $X$ lifts to $W(\mathbb{F}) / p^{2}$, we will deduce that $C$ need only depend on $\operatorname{deg}(f)$ and on the $\ell$-adic Betti numbers of $X$.

Let $k$ be any field. If $X / k$ is a projective surface, we denote its Hodge numbers by $h^{i j}(X)=$ $\operatorname{dim} H^{j}\left(X, \Omega_{X}^{i}\right)$.

Lemma 3.1. If $X / k$ is a smooth projective surface of general type with specified Hodge numbers $h^{i j}(X)=$ $h^{i j}$, then there is a bound for the type of X which depends only on $h^{i j}$.

Proof. First, we prove the result under the additional assumption that $X$ is minimal. Since the Hodge numbers of $X$ are known, in particular one knows $\chi\left(X, \mathcal{O}_{X}\right)=\sum_{i}(-1)^{i} h^{0, i}(X)$ and $K_{X}^{2}=$ $12 \chi\left(X . \mathcal{O}_{X}\right)-\sum(-1)^{i+j} h^{i j}(X)$. Since $X$ is of general type, $5 K_{X}$ is very ample. Let $N=10 K_{X}^{2}+$ $\chi\left(\mathcal{O}_{X}\right)-1$. Then $\phi_{5 K_{X}}: X \rightarrow \mathbb{P}^{N}$ is birational onto its image $X_{0}$. The embedded surface $X_{0} \subset \mathbb{P}^{N}$ has Hilbert polynomial $h_{X_{0}}(T)=(25 / 2)\left(K_{X}^{2}\right) T^{2}-(5 / 2) K_{X}^{2} T+\chi\left(\mathcal{O}_{X}\right)$, and thus is of known type. Moreover, $X_{0}$ is normal, with at worst Du Val singularities corresponding to the contraction of $(-2)$ curves on $X$. The number $m$ of such curves may be bounded in terms of the Hodge numbers of $X$ [8, p. 614]. Since $X$ is obtained from $X_{0}$ by at most $m$ blowups, $X$ has known type.

Finally, we prove the result for arbitrary smooth projective surfaces of general type. If $X$ is such a surface, and if $\pi: X \rightarrow \bar{X}$ is the blowing-down of a $(-1)$-curve, then $h^{1,1}(X)=h^{1,1}(\bar{X})+1$, while $h^{i j}(X)=h^{i j}(\bar{X})$ for all other $(i, j)$. Therefore, $X$ differs from its minimal model $X_{\min }$ by at most $h^{1,1}(X)$ blow-ups, and the Hodge numbers of $X_{\min }$ are known. Since a variety obtained by a bounded number of blowups from a variety of known type is again of known type, $X$ has known type.

Corollary 3.2. Given nonnegative integers $b_{1}, b_{2}$ and $b_{3}$ and a natural number $n$, there exists an effective constant $C$ so that the following holds. Let $X$ be a smooth, projective, geometrically irreducible surface over a finite field $\mathbb{F}$. Suppose that $X$ is of general type and admits a flat lifting to $W(\mathbb{F}) / p^{2}$, and that $\operatorname{dim} H^{i}\left(X, \mathbb{Q}_{\ell}\right)=b_{i}$ for $1 \leq i \leq 3$. Then $(X / \mathbb{F}, C, n)$ satisfies (1.1).

Proof. The hypothesis that $X$ lifts modulo $p^{2}$ implies that the Hodge to deRham sequence for $X$ degenerates [4]; this, combined with the comparison theorem between étale and deRham cohomology, implies that $\sum_{r=0}^{i} h^{i, i-r}(X)=b_{i}$ for each $i$. Therefore, there are only finitely many possibilities for the Hodge numbers of $X$, and thus for the type of $X$ (Lemma 3.1). The result now follows from Theorem 2.5.

Similar results are possible for surfaces which are not necessarily of general type. Here is one example. 
Corollary 3.3. Given a natural number $n$, there exists a constant $C$ so that the following holds. Let $\mathbb{F}$ be a finite field of odd characteristic, and let $X / \mathbb{F}$ be an Enriques surface. Then $(X / \mathbb{F}, C, n)$ satisfies (1.1).

Proof. By [1, Introduction], $X_{\overline{\mathbb{F}}}$ admits an étale double cover which is the intersection of three quadrics in $\mathbb{P}^{5}$; therefore, $X_{\overline{\mathbb{F}}}$ is of known type. For each scheme $Z$ which arises in the proof of Theorem 2.5, $Z_{\overline{\mathbb{F}}}$ is of known type. Therefore, the conclusion of 2.5 applies to $X$.

\section{EXAMPLES}

Most known examples of exceptional covers involve curves, especially the projective line. Higherdimensional examples tend to involve special varieties, such as abelian varieties or projective spaces. While exceptional covers are indeed rare, in this section we show that there actually exist infinitely many exceptional covers of each dimension over a given finite field. See also forthcoming work of Lenstra, Moulton and Zieve.

Theorem 2.5 (like its antecedents in [6]) states that if a finite field is sufficiently large relative to the topology of two varieties, then a cover is injective on rational points if and only if it is surjective. We give examples showing that this fails if the field is not sufficiently large.

Throughout this section, let $\mathbb{F}=\mathbb{F}_{q_{0}}$ be a finite field of cardinality $q_{0}$.

Proposition 4.1. Let $Y / \mathbb{F}$ be a smooth projective geometrically irreducible variety.

(a) There exists an exceptional cover $f_{\mathrm{ex}}: X_{\mathrm{ex}} \rightarrow Y$.

(b) There exists a cover $f_{\text {surj }}: X_{\text {surj }} \rightarrow Y$ such that $X_{\text {surj }}(\mathbb{F}) \rightarrow Y(\mathbb{F})$ is surjective but not injective.

(c) Suppose $Y(\mathbb{F})$ is nonempty. There exists a cover $f_{\mathrm{inj}}: X_{\mathrm{inj}} \rightarrow Y$ such that $X_{\mathrm{inj}}(\mathbb{F}) \rightarrow Y_{\mathrm{inj}}(\mathbb{F})$ is injective but not surjective.

Each of $X_{\mathrm{ex}}, X_{\mathrm{surj}}$ and $X_{\mathrm{inj}}$ is a projective smooth geometrically irreducible variety, and each of $f_{\mathrm{ex}}, f_{\mathrm{surj}}$ and $f_{\text {inj }}$ is a finite, generically étale surjective morphism.

We present constructions after recalling some of the technology for producing space-filling and space-avoiding varieties developed by Poonen in [9].

Lemma 4.2. Let $f: X \rightarrow Y$ be a surjective morphism of smooth, projective geometrically irreducible varieties over $\mathbb{F}$ which is smooth over an open subset of $Y$ containing $Y(\mathbb{F})$. Then there exists a smooth projective geometrically irreducible subvariety $Z \subset X$ such that $Z(\mathbb{F})=X(\mathbb{F})$, and $Z \hookrightarrow X \rightarrow Y$ is a surjective morphism which is étale over an open subset of $Y$ containing $Y(\mathbb{F})$.

Proof. Fix an embedding $X \hookrightarrow \mathbb{P}^{M}$. By induction on $r:=\operatorname{dim} X-\operatorname{dim} Y$, it suffices to show that there is a hypersurface $H \subset \mathbb{P}^{M}$ such that $Z:=H \cap X$ is smooth, projective and geometrically irreducible, $Z(\mathbb{F})=X(\mathbb{F})$, and $\left.f\right|_{Z}: Z \rightarrow Y$ is smooth of relative dimension $r-1$ over an open subset of $Y$ which contains $Y(\mathbb{F})$.

We follow the proof of [9. Thm. 3.3], and describe suitable hypersurfaces in terms of local tangency conditions. In this description, all intersections are scheme-theoretic, and the empty scheme is smooth of any dimension. Let $S=Y(\mathbb{F})$; if $S$ is empty, let $S$ consist of a point $Q \in Y(\overline{\mathbb{F}})$ such that $\left.f\right|_{f^{-1}(Q)}$ is smooth.

For each $P \in X(\mathbb{F})$, choose a codimension one subspace $V_{P} \subset T_{P, \mathbb{P} M}$ such that $V_{P} \cap T_{P, X}$ has codimension one in $T_{P, X}$, and the induced map $(d f)_{P}:\left(V_{P} \cap T_{P, X}\right) \rightarrow T_{f(P), Y}$ is surjective. Consider the problem of finding a hypersurface $H \subset \mathbb{P}^{M}$ such that for each $P \in X(\mathbb{F}), P \in H$ and $T_{P, H}=$ $V_{P}$; and for each other closed point $P$ of $X, H$ and $H \cap X$ are smooth of dimensions $M-1$ and 
$\operatorname{dim} X-1$, respectively, at $P$. Refine this problem by insisting that for each closed point $P$ of $X$ and each $Q \in S$, the intersection $H \cap X \cap f^{-1}(Q)$ is smooth of dimension $r-1$ at $P$.

Then [9. Thm. 1.3] guarantees the existence of a smooth geometrically irreducible hypersurface which satisfies these conditions. Choose such a hypersurface $H$, and let $Z=H \cap X$. Then $Z$ is smooth, $Z(\mathbb{F})=X(\mathbb{F})$, and the morphism $Z \hookrightarrow X \rightarrow Y$ is generically smooth of relative dimension $r-1$, and in particular smooth over each $\mathbb{F}$-rational point of $Y$. A dimension count shows the morphism is dominant, and thus surjective.

There is a point-avoiding complement to Lemma 4.2

Lemma 4.3. Let $f: X \rightarrow Y$ be a surjective morphism of smooth, projective geometrically irreducible varieties over $\mathbb{F}$ which is smooth over an open subset of $Y$ containing $Y(\mathbb{F})$. Then there exists a smooth projective geometrically irreducible subvariety $Z \subset X$ such that $Z(\mathbb{F})$ is empty, and $Z \hookrightarrow X \rightarrow Y$ is a surjective morphism which is étale over an open subset of $Y$ containing $Y(\mathbb{F})$.

Proof. This is a relative version of [9, Cor. 3.6]. The proof is the same as that of Lemma 4.3, except that in the local conditions we insist that the hypersurface avoid each point of $X(\mathbb{F})$.

With these results secured, construction of examples is straight-forward.

Proof of Proposition 4.1 For part (a), let $Y_{0}=\operatorname{Spec} B$ be an open affine subvariety of $Y$. Fix a prime $\ell$ relatively prime to $q_{0}-1$, and let $u \in B$ be an element of some system of uniformizing parameters for $Y_{0}$. Let $A=B[x] /\left(x^{\ell}-u\right)$, and let $X_{0}=\operatorname{Spec} A$. Then $X_{0}$ is geometrically irreducible (since $x^{\ell}-u$ is irreducible over $B \otimes \overline{\mathbb{F}}$ ) and smooth (by the Jacobian criterion). Let $\mathbb{F}_{q} / \mathbb{F}$ be any finite extension with $\operatorname{gcd}(q-1, \ell)=1$, and suppose $Q \in Y_{0}\left(\mathbb{F}_{q}\right)$. Then there is a unique solution in $\mathbb{F}_{q}$ to the equation $x^{\ell}=u(Q)$, and $X_{0}\left(\mathbb{F}_{q}\right) \rightarrow Y_{0}\left(\mathbb{F}_{q}\right)$ is bijective. Since this is true for arbitrarily large $q$, the cover $X_{0} \rightarrow Y_{0}$ is exceptional. Let $X$ be the normalization of $Y$ in $\operatorname{Frac}(A)$; it, too, is smooth. The geometric characterization of exceptionality makes it clear that being exceptional is a birational property, and so $X \rightarrow Y$ is exceptional.

For (b), let $V$ be a smooth projective geometrically irreducible variety such that $|V(\mathbb{F})| \geq 2$; concretely, one may take $V=\mathbb{P}^{1}$. Then the product $Y \times V$ is again smooth projective and geometrically irreducible, and the projection $Y \times V \rightarrow Y$ is smooth. Moreover, $(Y \times V)(\mathbb{F}) \rightarrow Y(\mathbb{F})$ is surjective but not injective. By Lemma 4.2, there is a smooth projective geometrically irreducible subvariety $\widetilde{X}_{\text {surj }} \subset Y \times V$ such that $\widetilde{X}_{\text {surj }}(\mathbb{F})=(Y \times V)(\mathbb{F})$, and $\left.f\right|_{\widetilde{X}_{\text {surj }}}$ is étale over each element of $Y(\mathbb{F})$. Consider the Stein factorization $\tilde{X}_{\text {surj }} \stackrel{s}{\rightarrow} X_{\text {surj }} \stackrel{t}{\rightarrow} Y$ of the projective morphism $\left.f\right|_{\widetilde{X}_{\text {surj }}}$. The morphism $t$ is finite and generically étale. Moreover, since $\tilde{X}_{\text {surj }} \rightarrow Y$ is finite over $Y(\mathbb{F})$, the birational morphism $s$ induces a bijection $\tilde{X}_{\text {surj }}(\mathbb{F}) \stackrel{\sim}{\rightarrow} X_{\text {surj }}(\mathbb{F})$. Therefore, $X_{\text {surj }} \rightarrow Y$ is finite and generically étale, and $X_{\text {surj }}(\mathbb{F}) \rightarrow Y(\mathbb{F})$ is surjective but not injective.

The proof of (c) is similar, except that we use Lemma 4.3 to construct a suitable subvariety $\widetilde{X}_{\text {inj }} \subset Y \times V$ with $\widetilde{X}_{\text {inj }}(\mathbb{F})$ empty. Again, the Stein factorization $\widetilde{X}_{\text {inj }} \rightarrow X_{\text {inj }} \rightarrow Y$ yields a finite cover of $Y$, and $\widetilde{X}_{\text {inj }}(\mathbb{F}) \stackrel{\sim}{\rightarrow} X_{\text {inj }}(\mathbb{F})$. If $Y(\mathbb{F})$ is nonempty, then $X_{\text {inj }}(\mathbb{F}) \rightarrow Y(\mathbb{F})$ is injective but not surjective.

Remark 4.4. The construction of the "space-filling" variety $X_{\text {surj }} \subset Y \times V$ in Proposition 4.1.(b) depends on $\mathbb{F}$; the equality of sets $X_{\text {surj }}(\mathbb{F})=(Y \times V)(\mathbb{F})$ is only possible if $q_{0}$ is small relative 
to the Betti numbers of $X_{\text {surj. }}$ Similarly, in 4.1.(c), the variety $X_{\text {inj }}$ acquires rational points over sufficiently large extensions of $\mathbb{F}$. While there is no reason to believe that the constant $C$ in Theorem 2.5 is optimal, these examples indicate that the equivalences in $\left(^{*}\right)$ cannot hold for $\mathbb{F}$ itself, but only for sufficiently large extensions.

\section{REFERENCES}

[1] F. R. Cossec. Projective models of Enriques surfaces. Math. Ann., 265(3):283-334, 1983.

[2] P. Deligne. Cohomologie étale. Springer-Verlag, Berlin, 1977. Séminaire de Géométrie Algébrique du Bois-Marie SGA $4 \frac{1}{2}$, Avec la collaboration de J. F. Boutot, A. Grothendieck, L. Illusie et J. L. Verdier, Lecture Notes in Mathematics, Vol. 569.

[3] P. Deligne. La conjecture de Weil. II. Inst. Hautes Études Sci. Publ. Math., (52):137-252, 1980.

[4] P. Deligne and L. Illusie. Relèvements modulo $p^{2}$ et décomposition du complexe de de Rham. Invent. Math., 89(2):247-270, 1987.

[5] M. Fried. On a theorem of MacCluer. Acta Arith., 25:121-126, 1973/74.

[6] R. M. Guralnick, T. J. Tucker, and M. E. Zieve. Exceptional covers and bijections on rational points. Int. Math. Res. Notices, 2007(4):19 pages, 2007.

[7] E. Kowalski. The large sieve, monodromy and zeta functions of curves. J. Reine Angew. Math., 601:29-69, 2006.

[8] D. Mumford. The canonical ring of an algebraic surface. Ann. Math. (2), 76:612-615, 1962. Appendix to O. Zariski, The theorem of Riemann-Roch for high multiples of an effective divisor on an algebraic surface.

[9] B. Poonen. Bertini theorems over finite fields. Ann. of Math. (2), 160(3):1099-1127, 2004.

E-mail address: j. achter@colostate.edu

Department of MATHEMATICS, COLORAdo StATE University, Fort COLlins, CO 80523 